\title{
INCREASE IN NEUTRON REFLECTIVITY FROM BIG Cu AND CoFe (8\%AT.) ALLOY SINGLE CRYSTAL PLATES RESULTING FROM COLD HAMMERING OF THE PLATES
}

\author{
M. ŚLEPoWrońsKI, S. BeDNARSKI, M. WIECZoRKowSKI \\ AND E.F. MALISZEWSKI
}

Institute of Atomic Energy, 05-400 Świerk-Otwock, Poland

In the paper changes of neutron reflectivity of big $\left(120 \times 70 \times 10 \mathrm{~mm}^{3}\right)$ $\mathrm{Cu}$ single crystalline monochromators and CoFe (8\%at.) single crystalline, polarising monochromators after cold hammering of the starting plates, are described. An almost three times higher number of reflected neutrons (total reflectivity) and about $20 \%$ higher intensity in peak maximum were observed, for $\mathrm{Cu}$ plates (measured in reflection from (220) plane)), while the mosaic's spread was about 2 times higher than the one measured for a non-treated plate. In the case of CoFe, $3 \mathrm{~mm}$ thick plates, a two times higher number of reflected neutrons and almost $60 \%$ higher intensity in peak maximum were observed (measured in transmission - from (200) plane) after hammering, while the mosaic's spread increase was rather small (about $15 \%)$.

PACS numbers: 06.90.+v, 81.40.-z

\section{Introduction}

The neutron spectrometry is still an intensity limited technique. For this reason, many attempts have been made to increase the intensity of the neutron beam. There are two possible methods of such an increase:

first - an increase in the reactor power (a very expensive way) and second - an increase in the neutrons reflectivity of the monochromators used in spectrometers and also focusing the neutron beam.

The intensity of the neutron beam reflected from a given crystal and intensity in peak maximum depend (among other factors) on the mosaic spread (FWHM). Additionally, for some experiments the specified FWHM of the crystals is required.

Many different methods are used to increase the reflectivity of as-grown crystals:

- using wafer stacks [1], 
- using gradient crystals [2],

- cold and hot pressing of the crystals $[3,4]$ and other methods.

In our laboratory, the cold hammering method [4] was used to increase the reflectivity of small single crystalline plates of $\mathrm{Cu}$. Presently, after some modifications of our equipment, this method is used to increase the reflectivity of big single crystalline plates of $\mathrm{Cu}$ and also $\mathrm{CoFe}(8 \%$ at.) alloy.

\section{Experimental procedure}

Big $\mathrm{Cu}$ and $\mathrm{CoFe}$ (8\%at.) single crystals are grown in our laboratory by vertical Bridgman technique in own construction resistance furnaces [5]. Standard parameters of as-grown crystals are as follows:

a) Cu-cylinders of about $120 \mathrm{~mm}$ in diameter and about $200 \mathrm{~mm}$ long; the measured average mosaic spread (FWHM) is $15 \pm 3$ minutes of arc,

b) $\mathrm{CoFe}$ (8\%at.)-cylinders of about $70 \mathrm{~mm}$ in diameter and $120 \div 130 \mathrm{~mm}$ long; the measured average mosaic spread (FWHM) in most of the cases is $13 \pm 3$ minutes of arc.

$\mathrm{X}$-ray Laue back reflection method is used for as grown crystals orientation. Big crystals are cut into the oriented plates using electroerosion spark machine. Using such cutting technique, one introduces a much smaller number of defects than in the case of mechanical cutting. The damage layer of the crystal is in this case thinner (tenth of a millimeter), giving us a mosaic (FWHM) lower by 3 or even more minutes of arc. After cutting, plates are mechanically polished and chemically etched.

Deformation of the plates is provided by hammering of the planar samples which are simultaneously exposed to the stress from 3.5 up to $4.0 \mathrm{kG} / \mathrm{cm}^{2}$, in an especially prepared equipment. Crystals are hammered at room temperature.

Neutron measurements were performed using a triple axis neutron crystal spectrometer. The reflecting planes of the $\mathrm{Zn}$ monochromator, of the measured sample and of the $\mathbf{Z n}$ analyser were placed in parallel arrangement, respectively. The measurements were performed in Bragg reflection position.

$\mathrm{Cu}$ plates were measured in reflection geometry (reflection from (220) plane) while CoFe plates were measured in transmission geometry (reflection from (200) plane). The neutron wavelength was $1.512 \AA$.

The sizes of the prepared samples were:

a) big Cu plates $-110 \times 100 \times 8 \mathrm{~mm}^{3}$ with the reflecting big face $110 \times$ $100 \mathrm{~mm}^{2}$ parallel to (110) crystallographic plane (appointed for one plate monochromators),

b) Cu slabs $-110 \times 14 \times 8 \mathrm{~mm}^{3}$ with the reflecting big face $110 \times 14 \mathrm{~mm}^{2}$ parallel to (110) crystallographic plane (useful for multi-plate monochromator construction) and

c) $\mathrm{CoFe}\left(8 \%\right.$ at.) plates $-35 \times 35 \times 3 \mathrm{~mm}^{3}$ with the big face $35 \times 35 \mathrm{~mm}^{2}$ parallel to (110) crystallographic plane and reflecting $35 \times 3 \mathrm{~mm}^{2}$ face parallel to (100) crystallographic plane (useful as polarising monochromators). 
The different sizes of the samples were determined by their potential application. This variety of sizes was also helpful for us to obtain a more differentiated energy transfer to the samples during the hammering process.

The estimated energies, transferred to the sample during hammering, are shown in Table.

TABLE

The estimated energies transferred to the samples and the constant stress values used during hammering of single crystalline $\mathrm{Cu}$ and $\mathrm{CoFe}(8 \%$ at.) plates.

\begin{tabular}{l|c|c}
\hline \hline \multicolumn{1}{c|}{ Sample } & Energy density $\left[\mathrm{J} / \mathrm{cm}^{3}\right]$ & Stress $\left[\mathrm{kG} / \mathrm{cm}^{2}\right]$ \\
\hline Big Cu plates of the size & $\approx 70$ & 3.50 \\
$110 \times 100 \times 8 \mathrm{~mm}^{3}$ & & \\
\hline Cu slabs of the size & $\approx 450$ & 3.50 \\
$110 \times 14 \times 8 \mathrm{~mm}^{3}$ & $\approx 540$ & 4.00 \\
\hline CoFe $(8 \%$ at.) plates of the & $\approx 1600$ & 2.45 \\
size $35 \times 35 \times 3 \mathrm{~mm}^{3}$ & $\approx 2500$ & 4.00
\end{tabular}

\section{Results}

The results of the small $\left(70 \mathrm{~J} / \mathrm{cm}^{2}\right)$, succeeding energy transfers during hammering of the big Cu plates are shown in Figs. 1 and 2 . The $35 \%$ increase in the mosaic value (FWHM) - from 14.04 minutes of arc (for not hammered plate)

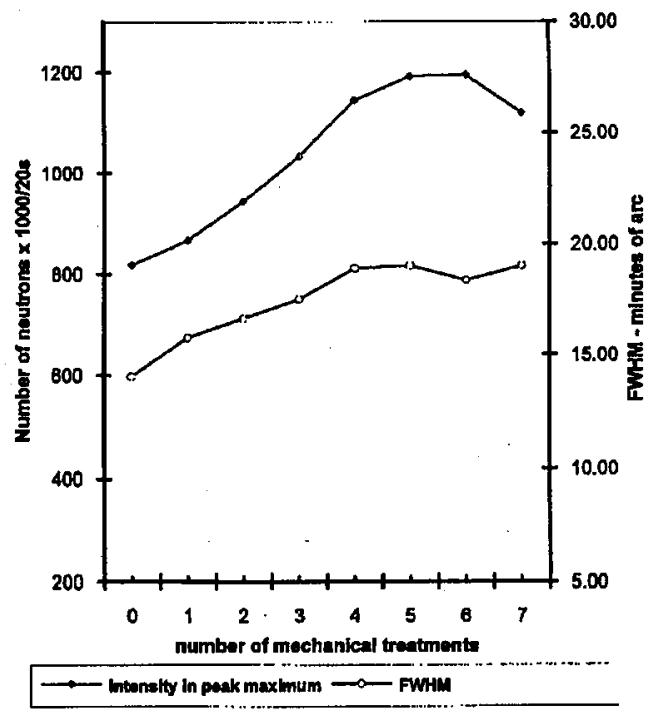

Fig. 1. Mosaic (FWHM) and intensity in peak maximum changes measured for $\mathrm{Cu}$ plate of the size $\left(110 \times 100 \times 8 \mathrm{~mm}^{3}\right)$ after succeeding mechanical treatments, $(220)$ plane - in reflection; unpolarised beam, neutron wavelength $1.512 \AA$. 


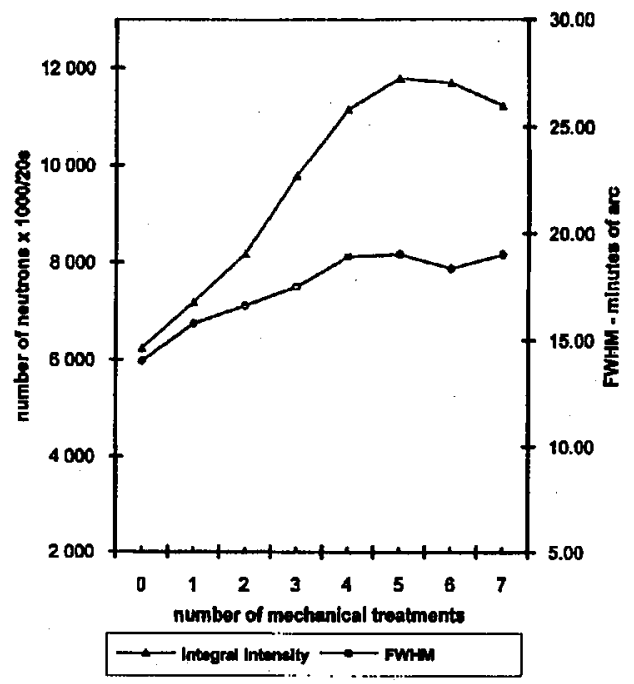

Fig. 2. Mosaic (FWHM) and integral intensity changes measured for $\mathrm{Cu}$ single crystalline plate of the size $\left(110 \times 100 \times 8 \mathrm{~mm}^{3}\right)$ after succeeding mechanical treatments, (220) plane - in reflection; unpolarised beam, neutron wavelength $1.512 \AA$.

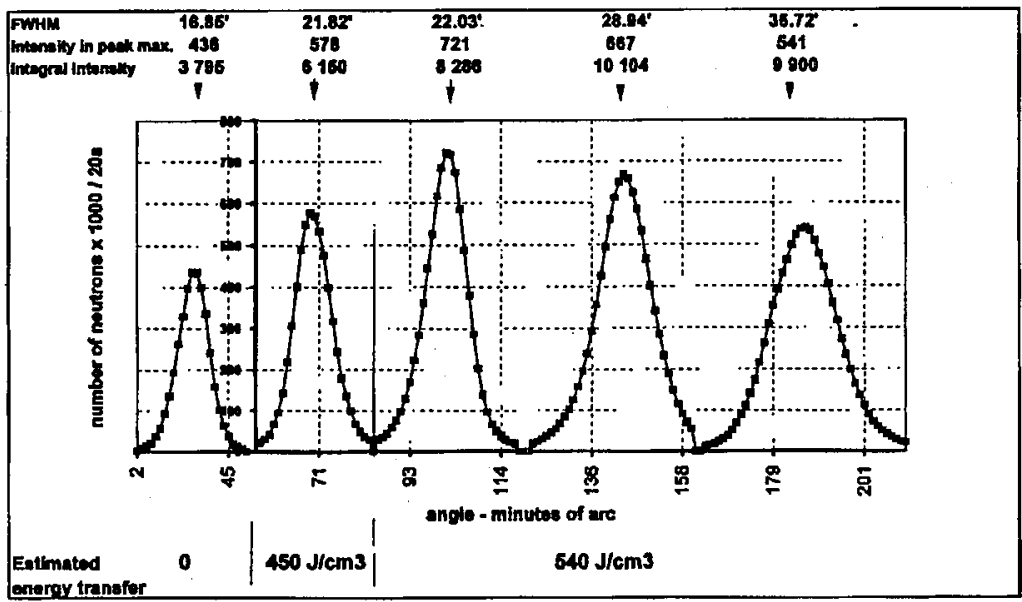

Fig. 3. Rocking curves measured for $\mathrm{Cu}$ single crystal plates before and after different mechanical treatment. (220) plane - in reflection. Plates of the size $110 \times 14 \times 8 \mathrm{~mm}^{3}$ were cut off from the same $\mathrm{Cu}$ crystal; unpolarised beam, neutron wavelength $1.512 \AA$.

up to 19.01 minutes of arc (for a deformed sample) - was measured while the measured intensity in peak maximum and integral intensity increased by $37 \%$ and $80 \%$, respectively.

In the case of $\mathrm{Cu}$ slabs, for which an almost one order higher energy transfer occurred, the FWHM value increased from $29 \%$ to over $100 \%$ of initial value, 


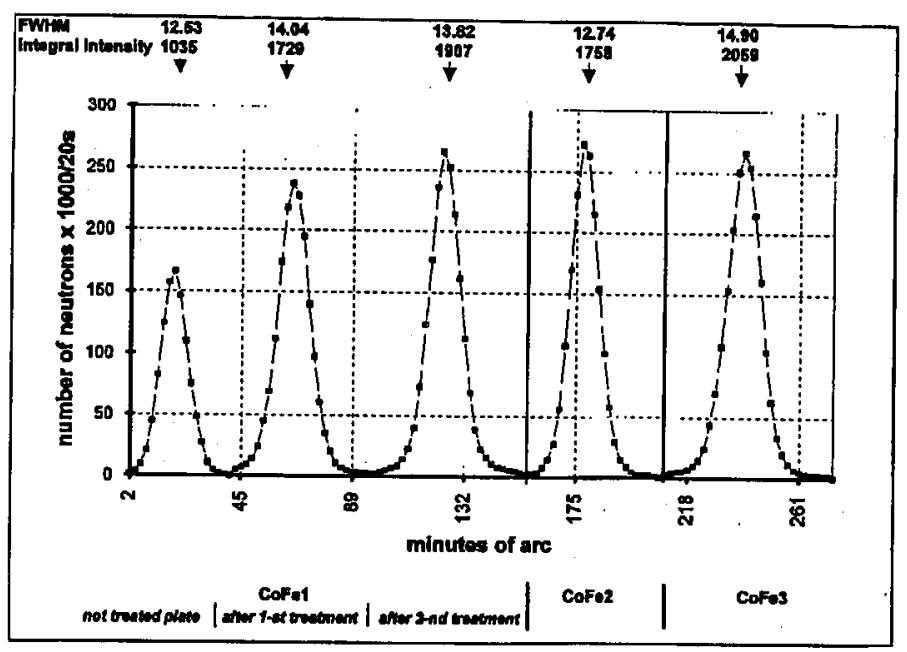

Fig. 4. Rocking curves measured for $\mathrm{CoFe}(8 \% \mathrm{at}$ ) plates, in transmission - reflection from (200) plane. Plates of the size $35 \times 35 \times 3 \mathrm{~mm}^{3}$; before and after different mechanical treatments; unpolarised beam, neutron wavelength $1.512 \AA$.

while the increase in the intensity in peak maximum from $24 \%$ up to $65 \%$ and integral intensity increase from $62 \%$ up to $166 \%$ of initial values were measured (see Fig. 3).

An effect of FWHM value decrease from 21 to 18 minutes of arc was observed for one of the big, hammered $\mathrm{Cu}$ plates after it was cut into some smaller slabs. This means that electroerosion cutting can decrease stresses in the previously deformed material and that the plates deformation should be the last applied process.

In the case of CoFe single crystal plates which are used as neutron polarizers, the neutrons reflectivity is one of the most important parameters, decisive for a crystal quality. Experimental results of hammering, obtained for these crystals are presented in Fig. 4. They are very interesting. The intensity in peak maximum and also the integral intensity measured for the deformed plates are in extremal values almost two times higher than those measured for the plate before hammering, while the measured differences in FWHM values do not exceed $20 \%$. The best results were obtained for $\mathrm{CoFe}_{2}$ sample which was annealed before hammering, at $1160^{\circ} \mathrm{C}$ during 48 hours. The mosaic spread (FWHM) measured for this sample after deformation is only $2 \%$ higher than the one measured before its hammering, while the $64 \%$ increase in intensity in peak maximum was observed for this sample.

The influence of $\mathrm{CoFe}$ samples hammering on their polarizing properties is now measured and will be published in the near future.

\section{References}

[1] T. Vogt, L. Passell, S. Cheung, J.D. Axe, Nucl. Instrum. Methods Phys. Res. 338, 71 (1994).

[2] D. Dorner, Nucl. Instrum. Methods Phys. Res. 338, 33 (1994). 
[3] V. Wagner, P. Mikula, P. Lukas, Nucl. Instrum. Methods Phys. Res. 338, 53 (1994).

[4] R.E. Lechner, R.V. Wallpach, H.A. Graf, F.-J. Kasper, L. Mokrani, Nucl. Instrum. Methods Phys. Res. 338, 65 (1994).

[5] S. Bednarski, E. Moch, H. Piotrowski, K. Zając, L. Chełmicki, S. Wąsowski, in: Technologia Monokrysztatów, Proc. I Ogólnopolskie Seminarium Technologii Monokrysztatów, Szklarska Poręba (1972), Part 1, PWN, Warszawa 1974, p. 244 (in Polish). 